\title{
Wave-packet dynamics at the mobility edge in two- and three-dimensional systems
}

\author{
Bodo Huckestein \\ Institut für Theoretische Physik, Universität zu Köln, D-50937 Köln, Germany \\ Rochus Klesse \\ Institut für Theoretische Physik, Universität zu Köln, D-50937 Köln, Germany, \\ and Department of Condensed Matter Physics, Weizmann Institute of Science, 76100 Rehovot, Israel
}

(Received 4 May 1998; revised manuscript received 23 December 1998)

\begin{abstract}
We study the time evolution of wave packets at the mobility edge of disordered non-interacting electrons in two and three spatial dimensions. The results of numerical calculations are found to agree with the predictions of scaling theory. In particular, we find that the return probability $P(r=0, t)$ scales like $t^{-D_{2} / d}$, with the generalized dimension of the participation ratio $D_{2}$. For long times and short distances the probability density of the wave packet shows power law scaling $P(r, t) \propto t^{-D_{2} / d} r^{D_{2}-d}$. The numerical calculations were performed on network models defined by a unitary time evolution operator providing an efficient model for the study of the wave packet dynamics.
\end{abstract}

The time evolution of wave packets is intimately related to the transport properties of an electron system. If a wave packet does not spread unboundedly for long times the system is an insulator. On the other hand, if a wave packet spreads diffusively for long times the system has a finite conductivity characteristic of a metal. In a seminal paper Anderson observed that disorder can lead to the absence of diffusion 1 With the advent of the scaling theory of localizationet 4 it was realized that the existence of this Anderson transition between localized and metallic states depends essentially on the dimensionality and the symmetries of the system only. In one dimension any amount of disorder leads to localization of all states while in two dimensions for non-interacting electrons either strong magnetic field (af im the quantum Hall effect 50 ) or spin-orbit interaction 810 are necessary for the existence of extended states. Three-dimensional system generally show an Anderson transition as function of disorder strength and Fermi energy.

At the Anderson transition a system shows a behavior intermediate between localized and extended. A crucial assumption of the scaling theory ${ }^{3}$ is the finite value of the conductance $G$ of the system at the transition. The conductance of a $d$-dimensional hyper-cube of linear dimension $L$ is related to the conductivity $\sigma$ by Ohm's law $G=\sigma L^{d-2}$. The conductivity at the transition thus scales like $L^{2-d}$ and so does the diffusion coefficient $D$ according to the Einstein relation $\sigma=e^{2} \rho D$, with the density of states $\rho$. The Anderson transition is not reflected in the density of states $\rho$ which is finite through the transition 1 . Accordingly, the diffusion coefficient is finite at a two-dimensional transition while there is no diffusive behavior in three dimensions.

Apart from this scale dependence of the diffusion coefficient a distinguishing feature of the Anderson tran- sition is the emergence of multifractal correlations of the wavefunction amplitudes, 11 13. The corresponding density-density correlator can be expressed in terms of a wave vector- and frequency-dependent diffusion coefficient $D(q, \omega)$, which in the DC limit becomes the diffusion coefficient discussed in the previous paragraph. For short distances and low frequencies, however, this diffusion coefficient reflects the strong amplitude fluctuations within single eigenfunctions 214 . It should be noted that multifractal correlations are also present away from the Anderson transition on scales smaller than the localization length 15.16 .

The scaling form of the diffusion coefficient $D(q, \omega)$ has been discussed previously in the literature 14 17 20. In this paper we study directly the time evolution of wave packets. The physical information contained in both quantities is the same. The advantage of our approach is that it obviates the need for a diagonalization of the Hamiltonian. Instead the time evolution operator is applied repeatedly. For systems defined by a Hamiltonian $H$ this is not necessarily easier to do as the time evolution operator $U=\exp (-i H t / \hbar)$ is an exponential of the Hamiltonian and the kinetic and potential terms in the Hamiltonian do not commute in general complicating the calculation of the exponential. To circumvent this problem decompositions of the exponential hyebeen devised 21 and applied to the problem at hand 22 24. On the other hand, for systems defined by a unitary operator that can be interpreted as a time evolution operator the dynamics of wave packets is the most natural and convenient quantity to study. A class of systems that falls into this category are the network models introduced by Shapiro 25 and Chalker and Coddington26. A network model is characterized by a unitary operator that describes the scattering of wavefunction amplitudes at the nodes of a network. Net- 
work models have been constructed and studied for twoand three-dimensional systems unithorthonal, symplectic, or chiral symmetry 27 30,10.31. The unitary operator of a network model can be interpreted as a discrete time evolution operator where a time step corresponds to the typical time of a scattering event 32 , 33 .

The spreading of wave packets has also been discussed in connection with the nature of the spectrum of the system. Ketzmerick et al. found that the spreading of wave packets is determined by the multifractal dimensions $\tilde{D}_{2}$ and $D_{2}$ of the local spectrum and the eigenfunctions, respectively 34 . While in general these dimensions are independent quantities characterizing a system, they are simply related in disordered system, $D_{2}=d \tilde{D}_{2}$, since the global density of states is scale invariant and-connects length and energy scales via the dimension $d^{29}$. In this paper we will restrict our discussion to the case of the Anderson transition.

After summarizing the scaling behavior of the probability density of a wave packet, we present the results of numerical simulations for two- and three-dimensional network models and show that they are in accordance with the predictions of scaling theory. In particular, we calculate the return probability to the origin of the wave packet, and the shape of the wave packet for long times and short distances. We conclude the paper with a discussion of the results. The scaling forms for the shape and moments of the wave packet as well as numerical results for the moments and preliminary results for the shape have been presented in 35 .

We now summarize the predictions of scaling theory for characteristic features of a wave packet. For the derivation we refer the reader to 35 and references therein.

Due to the occurence of multifractal eigenfunction fluctuations at the localization-delocalization transition, the shape of a wave packet differs strongly from the diffusive gaussian form. The long time, short distance behavior, $r^{d} \ll t / \hbar \rho$ with $\rho$ the density of states, of $P(r, t)$ is a power law 34 ,

$$
P(r, t) \propto t^{-D_{2} / d} r^{D_{2}-d} .
$$

The exponent $D_{2}$ is the generalized dimension of the inverse participation ratio. For multifractal eigenstates it is smaller than the space dimension $d$. The term short distance has to be understood in the sense of $r^{d} \ll t / \hbar \rho$. The distances on which Eq. (1) holds are large compared to any microscopic scale and correspond for $t \rightarrow \infty$ to the limit $r \rightarrow \infty$. For long times Eq. (1) thus describes the bulk of the wave packet up to an exponentially small tail.

From eq. (11) it follows that the return probability to the starting point of the wave packet $P(r=0, t)$ decays with time as

$$
P(r=0, t) \propto t^{-D_{2} / d} .
$$

This is the first quantity that we turn our attention to. It is the simplest quantity that reflects the multifractality of the eigenstates at the mobility edge. From

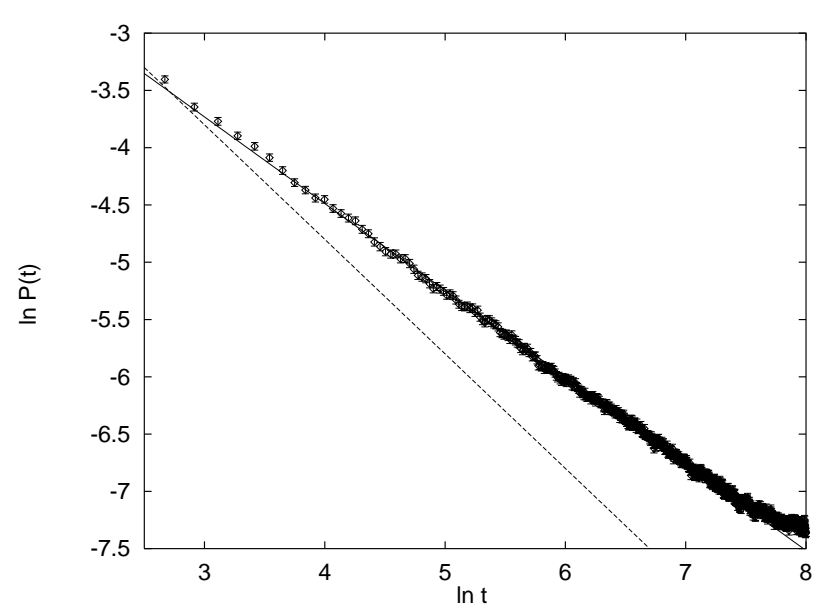

FIG. 1. Return probability for a wave packet at the quantum Hall critical point. The solid line is a best fit to the data with slope $D_{2} / 2=0.76 \pm 0.03$. The dashed line shows the classical behavior $p(t) \propto t^{-1}$.

Fig. (11) we get the exponent $D_{2} / 2$ of the return probability to be $0.76 \pm 0.03$, somewhat smaller but still in agreement with the value $0.81 \pm 0.02$ obtained by Huckestein and Schweitzer for the quantum Hall transition in a tight-binding mode 19 . In the present calculation, 400 realizations of the disorder were averaged for a system of $100 \times 100$ scatterers.

For a three-dimensional system the exponent $D_{2} / 3$ of the critical power law differs even more strongly from the diffusive value of $3 / 2$. The system that we studied is a stack of Hantum Hall networks coupled in the third dimension 28,29. For vanishing inter-layer coupling the system shows the quantum Hall transition of the isolated two-dimensional systems, while for finite coupling the system shows a three-dimensional Anderson transition with localized and non-localized regimes separated by mobility edges.

The return probability for the three-dimensional network model at the Anderson transition is shown in Fig. (2). For the time interval from 400 to 4900 time steps we obtain a power law with an exponent $D_{2} / 3=0.43 \pm$ 0.04. For shorter times a crossover to two-dimensional behavior is observable in our data. Its origin is the strongly anisotropic character of our system of coupled two-dimensional networks. The coupling in the third dimension is much weaker than in the planes so that threedimensional spreading is observed only after sufficiently long times. The data presented in Fig. (2) were calculated from 200 disorder realizations of a system of $50^{3}$ scatterers.

When comparing our result $D_{2}=1.3 \pm 0.1$ with values published in the literature it should be kept in mind that our network model corresponds to a system with a magnetic field. For time reversal symmetric systems in the absence of magnetic fields somewhat larger valyes have been published previously: $1.7 \pm 0.312,1.45-1.836$, 


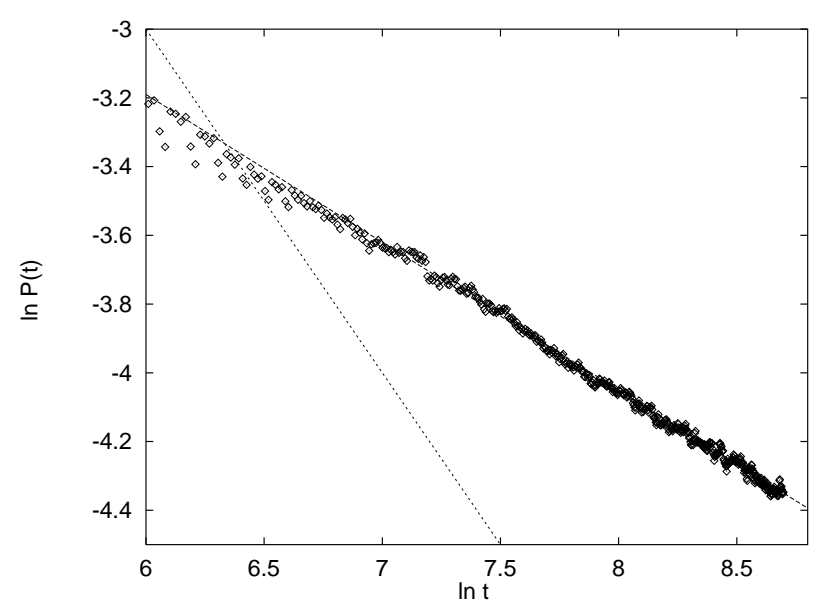

FIG. 2. Return probability for a wave packet at the Anderson transition in $d=3$. The solid line is a best fit to the data with slope $D_{2} / 3=0.43 \pm 0.04$. The dashed line shows the conventional behavior $p(t) \propto t^{-3 / 2}$.

$1.7 \pm 0.220,1.5 \pm 0.224$. The values obtained previously in the absence of time reversal symmetry do not differ significantly from these: $1.7 \pm 0.224, \approx 1.537,1.55 \pm 0.1538$. Again our value is somewhat but not significantly smaller than these values.

We now show that the bulk of a wave packet has a power law shape. For the two-dimensional quantum Hall system at the critical point the average probability density of the wave packet is plotted in Fig. (3) for different times $t$. The different curves have been rescaled by a factor of $t^{D_{2} / 2}$ in order to allow for a better comparison of densities at different times. The straight line shows the expected power law with exponent $D_{2}-2=-0.48$ where the value $D_{2}$ was taken from the fit to the return probability. The crossover from power law to exponential shape of the density distribution is seen to shift with increasing time to larger radii. 6 realizations of the disorder were averaged for a system of $300 \times 300$ scatterers.

Figure (14) shows the shape of the wave packet in a three-dimensional system of $50^{3}$ scatterers. At the critical point the power law with an exponent $D_{2}-3=-1.7$ is observed after 20000 time steps (solid boxes). In the metallic regime the density is practically constant over the whole system after 2400 time steps (open boxes). In the localized regime exponential localization is observed after 20000 time steps (triangles).

To summarize, we have studied the time evolution of wave packets at the mobility edge in disordered two- and three-dimensional electron systems. In order to characterize the wave packets we looked at the return probability and the shape of the density distribution. Due to the scale invariance of the critical systems these quantities show power law scaling. The exponents differ from the usual diffusive situation for two reasons. On the one hand, at the Anderson transition the conductance is scale invariant and not the conductivity or the diffusion coeffi-

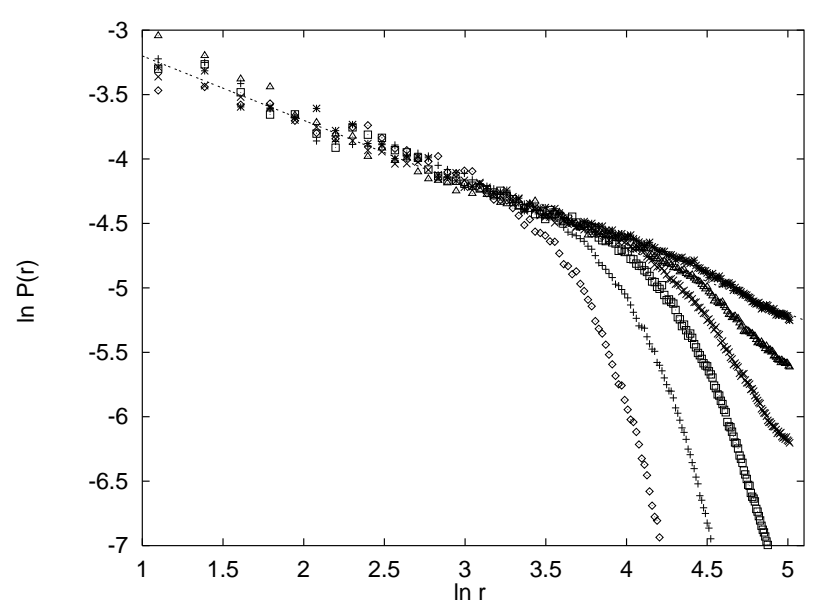

FIG. 3. Average density of wave packets at the quantum Hall critical point. The different curves correspond to different times (1500, 3000, 6000, 9000, 12000, 15000 time-steps). The different curves have been rescaled by an amount proportional to $t^{0.76}$.

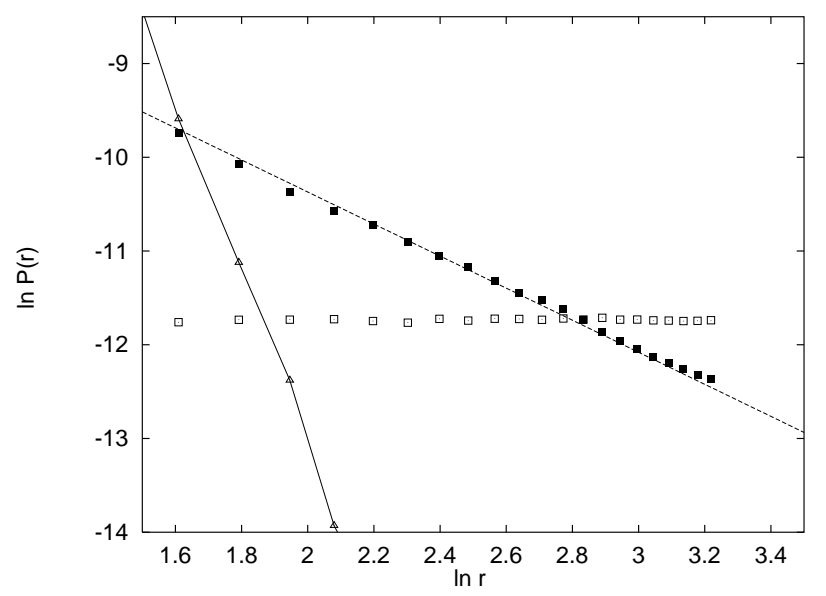

FIG. 4. Average density of wave packets in three-dimensional network models. Data are plotted: in the metallic regime after 2400 time steps $(\square)$, at the Anderson transition after 20000 time steps $(\boldsymbol{\square})$, and in the localized regime after 20000 time steps $(\triangle)$.

cient. As a result the spatial dimension $d$ enters where in the diffusive case the exponent 2 of the diffusion equation enters. On the other hand, the short distance long time behavior is governed by multifractal density correlations within single eigenfunctions leading to the replacement of the spatial dimension $d$ by the multifractal dimension $D_{2}$ of the inverse participation ratio.

We have confirmed the scaling laws and extracted the scaling exponents by numerical calculations. The calculation were performed for network models of disordered systems that are defined by a unitary network operator that serves as a discrete time evolution operator. This provides a very convenient and efficient way to study the 
time evolution of wave packets.

The time dependence of the return probability to the origin shows power law scaling with the exponent $-D_{2} / d$ involving the multifractal exponent $D_{2}$. Previously, this quantity was stydied in two- and three-dimensional tightbinding models 1920 , We obtain values for $D_{2}$ of $1.52 \pm 0.06$ and $1.3 \pm 0.1$ in two and three dimensions, respectively. These values are somewhat smaller, especially in three dimensions, than previously published values. At present, it is not clear whether this difference reflects genuinely different critical behavior in the systems or different strength of corrections to scaling. It is known that the two-dimensional network model has very small corrections to-sfaling compared to the random Landau matrix model 39.40 .

For long times $t$ and short distances, $r^{d} \ll t / \hbar \rho$, the shape of a wave packet becomes $t^{-D_{2} / d} r^{D_{2}-d}$. We observe this behavior in the two- and three-dimensional network models. At larger distances the crossover to exponential tails in the density distribution is observed.

Ketzmerick et al.34 obtained under quite general conditions the expression $P(r, t) \propto t^{-\tilde{D}_{2}} r^{D_{2}-d}$ for the shape of wave packets, where $\tilde{D}_{2}$ is the generalized dimension of the local density of states. These general results agree with our results for the Anderson transition since in the present case the generalized dimensions for the spectrum and the wave functions are not independent but proportional to each other, $D_{2}=d \tilde{D}_{2} 19,29$. The origin of this simplification is the scale invariance of the global density of states that allows to relate energy and length scales via a single relevant energy dependent length scale $L_{\omega} \propto \omega^{-1 / d}$.

This work was performed within the research program of the Sonderforschungsbereich 341 of the Deutsche Forschungsgemeinschaft.

${ }^{1}$ P. W. Anderson, Phys. Rev. 109, 1492 (1958).

${ }^{2}$ F. Wegner, Z. Phys. B 25, 327 (1976).

${ }^{3}$ E. Abrahams, P. W. Anderson, D. C. Licciardello, and V. Ramakrishnan, Phys. Rev. Lett. 42, 673 (1979).

${ }^{4}$ F. Wegner, Z. Phys. B 35, 207 (1979).

${ }^{5}$ The Quantum Hall Effect, Graduate Texts in Contemporary Physics, edited by R. E. Prange and S. M. Girvin (Springer, Berlin, 1987).

${ }^{6}$ M. Janssen, O. Viehweger, U. Fastenrath, and J. Hajdu, Introduction to the Theory of the Integer Quantum Hall Effect (VCH, Weinheim, 1994).

${ }^{7}$ B. Huckestein, Rev. Mod. Phys. 67, 357 (1995).

${ }^{8}$ S. Hikami, A. Larkin, and Y. Nagaoka, Prog. Theor. Phys. 63, 707 (1980).

${ }^{9}$ L. Schweitzer and I. Zharekeshev, J. Phys.: Condens. Matter 9, L441 (1997).

${ }^{10}$ R. Merkt, M. Janssen, and B. Huckestein, Phys. Rev. B
58, 4394 (1998).

${ }^{11}$ H. Aoki, J. Phys. C 16, L205 (1983).

${ }^{12}$ C. M. Soukoulis and E. N. Economou, Phys. Rev. Lett. 52, 565 (1984).

13 C. Castellani and L. Peliti, J. Phys. A 19, L429 (1986).

14 J. T. Chalker, Physica A 167, 253 (1990).

${ }^{15}$ V. Fal ko and K. Efetov, Europhys. Lett. 32, 627 (1995).

${ }^{16}$ V. Fal ko and K. Efetov, Phys. Rev. B 52, 17413 (1995).

17 J. T. Chalker and G. J. Daniell, Phys. Rev. Lett. 61, 593 (1988).

${ }^{18}$ Y. Huo, R. E. Hetzel, and R. N. Bhatt, Phys. Rev. Lett. 70, 481 (1993).

19 B. Huckestein and L. Schweitzer, Phys. Rev. Lett. 72, 713 (1994).

20 T. Brandes, B. Huckestein, and L. Schweitzer, Ann. Physik 5, 633 (1996).

${ }^{21}$ H. F. Trotter, Proc. Am. Math. Soc. 10, 545 (1959).

${ }^{22}$ T. Kawarabayashi and T. Ohtsuki, Phys. Rev. B 51, 10897 (1995).

${ }^{23}$ T. Kawarabayashi and T. Ohtsuki, Phys. Rev. B 53, 6975 (1996).

24 T. Ohtsuki and T. Kawarabayashi, J. Phys. Soc. Jpn. 66, 314 (1997).

${ }^{25}$ B. Shapiro, Phys. Rev. Lett. 48, 823 (1982).

${ }^{26}$ J. T. Chalker and P. D. Coddington, J. Phys. C 21, 2665 (1988).

${ }^{27}$ R. Klesse and M. Metzler, Europhys. Lett. 32, 229 (1995).

28 J. T. Chalker and A. Dohmen, Phys. Rev. Lett. 75, 4496 (1995).

29 B. Huckestein and R. Klesse, Phys. Rev. B 55, R7303 (1997).

${ }^{30}$ R. Klesse and M. Metzler, Phys. Rev. Lett. 79, 721 (1997).

${ }^{31}$ P. Freche, M. Janssen, and R. Merkt, Phys. Rev. Lett. 82, 149 (1999).

${ }^{32}$ R. Klesse, Ph.D. thesis, Universität zu Köln, (AWOSVerlag, Erfurt), 1996.

${ }^{33}$ R. Klesse and M. Metzler, to be published.

${ }^{34}$ R. Ketzmerick, K. Kruse, S. Kraut, and T. Geisel, Phys. Rev. Lett. 79, 1959 (1997).

${ }^{35}$ B. Huckestein and R. Klesse, Phil. Mag. 77, 1181 (1998).

${ }^{36}$ H. Grussbach and M. Schreiber, Phys. Rev. B 51, 663 (1995).

37 T. Terao, Phys. Rev. B 56, 975 (1997).

${ }^{38}$ L. Schweitzer, unpublished.

39 J. Eastmond and J.T. Chalker, unpublished.

${ }^{40}$ B. Huckestein, Phys. Rev. Lett. 72, 1080 (1994). 